\title{
Phenotypic and Genotypic Antibiotic Resistance of Salmonella from Chicken Carcasses Marketed at Ibague, Colombia
}

\section{-Author(s)}

\section{Cortes Vélez $D^{\prime}$}

Rodríguez V',1

Verjan García N',"l

Research Group in Poultry Science, Faculty of Veterinary Medicine, University of Tolima, Altos de Santa Helena A.A. 546, Ibague, Tolima Colombia.

"Immunobiology and Pathogenesis Research Group, Faculty of Veterinary Medicine, University of Tolima, Altos de Santa Helena A.A. 546, Ibague, Tolima.

\section{-Mail Address}

Corresponding author e-mail address Noel Verján García, Ph.D.

Department of Animal Health

University of Tolima, Faculty of Veterinary Medicine. Altos de Santa Helena, Ibagué, Colombia.

Tel: $\quad+57-8-27712$ 12, Ext 9216

Email: nverjang@ut.edu.co

\section{EKeywords}

Antimicrobial resistance, Poultry products, Serovars.

\section{ABSTRACT}

Salmonella enterica is responsible for alimentary toxic infections associated with the consumption of contaminated poultry products and the antimicrobial resistant patterns of Salmonella circulating in the Tolima region are currently unknown. To address this issue, both the phenotype and genotype antibiotic resistance patterns of 47 Salmonella isolated from raw chicken carcasses sold at the Ibague city were analyzed by the disc diffusion, microdilution and PCR assays. All $47 \mathrm{Salmonella} \mathrm{isolates} \mathrm{showed} \mathrm{resistance} \mathrm{to} \mathrm{five} \mathrm{or} \mathrm{more}$ antimicrobial agents. Resistance to Ampicillin (AMP), Amikacin (AMK), Gentamicin (GEN), Tobramycin (TOB), Cefazoline (CFZ), Cefoxitin (FOX), Nitrofurantoin (NIT), Trimethoprim-Sulfamethoxazole (SXT), Tetracycline (TET), Ciprofloxacin (CIP) and Enrofloxacin (ENR) was observed in $42.35 \%$ of Salmonella isolates. All tested S. Paratyphi B var Java isolates showed resistance to at least 12 antibiotics. S. Hvittingfoss showed resistance to 5 antibiotics, whereas $S$. Muenster showed resistance to seven antibiotics. Amplification of a number of antibiotic resistance genes showed that blaTEM (100\%) correlated well with resistance to Ampicilin and Cephalosporin, whereas aadB (87\%) correlated well with resistance to Aminoglycosides. It is concluded that Salmonella isolated from raw chicken meat marketed at Ibague showed MDR by both phenotypic and genotypic methods and they may represent an important threat to human health. Additional studies are needed to establish the relationship between antibiotic resistance in Salmonella from poultry products and clinical isolates.

\section{INTRODUCTION}

Salmonella enterica is a large group of Gram-negative bacteria that cause an infectious disease called salmonellosis. The subspecies enterica (I) groups the majority $(1547,60 \%)$ of serovars that affect human and domestic animals (Dekker \& Frank, 2015; Grimont \& Weill, 2007; OIE, 2012), and those serovars are classified as typhoidal and nontyphoidal Salmonella (NTS) (Sanderson \& Nair, 2013). Contaminated meat and poultry products such as commercial table eggs and raw chicken meat constitute the main sources of S. enterica (Ricke \& Calo, 2015). The disease in humans is characterized by a self-limiting gastrointestinal infection in immunocompetent patients, who usually develop fever, diarrhea and acute abdominal pain; however, it may progress into a life-threatening disease when the bacteria reach the bloodstream, particularly in young children, elderly and immunocompromised people (Mercado et al., 2012).

NTS, S. Enteritidis and S. Typhimurium are the most common serovars isolated from clinical cases of human salmonellosis (CDC, 2014; Hendriksen et al., 2011). S. Newport, S. Javiana, S.14,[5],12:i:-, S. Muenchen, S. Bareilly, S. Monevideo and S. Heildelberg, among 
others, are also associated in a smaller proportion with human infection (CDC, 2014; Chen et al., 2012). NTS might be responsible for about 80.3 million foodborne illnesses and 115,000 deaths each year in the world (Majowicz et al., 2010), while Typhoid, Paratyphoid and enteric fever cause 25 million infections and 200,000 deaths each year globally (Dekker \& Frank, 2015). Antibiotic treatment of salmonellosis is complicated because the microorganism under antibiotic pressure may select for virulence within the host (Diard et al., 2014), acquires tolerance and multiple drug resistance (MDR) phenotypes (fast-, moderate- and low-growing subsets) within host tissues (Claudi et al., 2014), and frequently incorporate new genetic material to resist the antibiotic selective pressure (Brown-Jaque et al., 2015). Salmonella isolated from food of animal origin shows higher rates of antimicrobial resistance (Chuanchuen \& Padungtod, 2009), which is promoted by the misuse or underuse of antimicrobials incorporated in feed to prevent infectious diseases and to promote bird growth, and those MDR microorganisms may disseminate very quickly with the rapid global food market .

In Colombia, limited information is available on the species of Salmonella circulating in poultry products, the serovars responsible for human infections as well as their antibiotic resistance patterns. Serovar Typhimurium variant 5 was isolated from human cases of salmonellosis in Paz del Rio, Boyacá (Díaz Osorio et al., 2014). Recently, our group isolated Salmonella Enteritidis and S. Shannon from laying-hen farms located in the Tolima region (Rodríguez et al., 2015a), and reported at least 14 different serovars of Salmonella from chicken carcasses sold at stores and supermarkets of Ibague (Rodriguez et al., 2015b), pointing out the importance of contaminated eggs and chicken meat as a potential source of human infection. In addition, manipulation, transportation and marketing of poultry products in most of the cases do not meet the standards of good manufacturing practices and instead they may promote contamination with Salmonella. In this study the antibiotic resistance patterns of Salmonella serovars isolated from chicken meat sold at stores and supermarkets in Ibague city were established.

\section{MATERIALS AND METHODS}

\section{Salmonella spp., isolates from chicken meat}

A total of 47 strains of Salmonella previously isolated from broiler carcasses marketed at Ibague, Colombia were used in this study. The Salmonella serovars were collected from a cross-sectional study conducted between February to May 2014 (Rodriguez et al., 2015b). The Salmonella isolates were thawed from glycerol stocks and streaked on TSA plates and incubated at $37^{\circ} \mathrm{C}$ for $24 \mathrm{hr}$.

\section{Phenotype of antibiotic resistance}

The Kirby-Bauer method (agar-disc diffusion) was used to evaluate the susceptibility of Salmonella to Chloramphenicol $(\mathrm{CHL}, 30 \mu \mathrm{g})$, Florfenicol (FFC, 30 $\mu \mathrm{g})$, Enrofloxacin (ENR, $5 \mu \mathrm{g})$, Norfloxacin (NOR, 10 $\mu \mathrm{g}$ ) and Fosfomycin (FOF, $50 \mu \mathrm{g}$ ), which are commonly used in veterinary medicine but they are not included in the automatized microdilution Phoenix TM (Becton Dickinson, Sparks, MD, USA) method. A bacterial suspension in Mueller-Hinton (Oxoid, Germany) agar was calibrated according to 0.5 McFarland scale of turbidity, and bacterial growth inhibition upon culture on plate at $37^{\circ} \mathrm{C}$ for $24 \mathrm{hr}$ was evaluated according to the guidelines of the Clinical Laboratory Standards Institute (CLSI, 2015).

Salmonella isolates were also subjected to an antimicrobial microdilution susceptibility test by using the BD Phoenix ${ }^{\mathrm{TM}}$ NMIC/ID-132 panels (Becton Dickinson, Sparks, MD, USA) and the categories established by The Clinical and Laboratory Standards Institute (CLSI, 2015). The antibiotics and concentration included in this assay were Amikacin (AMK, 8-32 $\mu \mathrm{g} / \mathrm{mL}$ ), Ampicillin (AMP, 4-16 $\mu \mathrm{g} / \mathrm{mL}$ ), AmpicillinSulbactam (SAM, 2/2 $\mu \mathrm{g} / \mathrm{mL}$ ), Aztreonam (ATM, 2-16 $\mu \mathrm{g} / \mathrm{mL}$ ), Cefazoline (CFZ, 2-16 $\mu \mathrm{g} / \mathrm{mL})$, Cefepime (FEP, 1-16 $\mu \mathrm{g} / \mathrm{mL})$, Cefoxitin (FOX, 4-16 $\mu \mathrm{g} / \mathrm{mL}$ ), Ceftriaxone (CRO, 2-32 $\mu \mathrm{g} / \mathrm{mL})$, Ciprofloxacin (CIP, 0.5-2 $\mu \mathrm{g} / \mathrm{mL})$, Ertapenem (ETP, 0.5-8 $\mu \mathrm{g} / \mathrm{mL}$ ), Gentamicin (GEN, 2-8 $\mu \mathrm{g} / \mathrm{mL}$ ), Imipenem (IPM, 1-8 $\mu \mathrm{g} / \mathrm{mL}$ ), Meropenem (MEM, 1-8 $\mu \mathrm{g} / \mathrm{mL}$ ), Nitrofurantoin (NIT, 16-64 $\mu \mathrm{g} /$ $\mathrm{mL}$ ), Piperacillin-Tazobactam (TZP, $0.5 / 16 \mu \mathrm{g} / \mathrm{mL}$ ), Tetracycline (TET, 2-8 $\mu \mathrm{g} / \mathrm{mL})$, Ticarcillin-Clavulanate (TIM, 4/32 $\mu \mathrm{g} / \mathrm{mL}$ ), Tobramycin (TOB, 2-8 $\mu \mathrm{g} / \mathrm{mL})$, and Trimethoprim-Sulfamethoxazole (SXT, 0.05/0.06). In this study, only absolutely but not intermediate resistant isolates of Salmonella were considered as resistant strains. Multi-drug resistant (MDR) strains of Salmonella were defined as those isolates that showed phenotype resistance to at least three or more classes of antibiotics. Escherichia coli ATCC 25922 was used as a reference strain.

\section{Genotype of antibiotic resistance}

Salmonellaisolateswereanalyzed by PCR to detect the presence of antibiotic resistance genes that are known to confer resistance to Ampicillin (blaTEM) with primer set blaTEM-F-5'-ATCAGTTGGGTGCACGAGTG-3 and blaTEM-R-5'- ACGCTCACCGGCTCCAGA-3', 
Chloramphenicol (catB) with primer set catB-F-5'CGGATTCAGCCTGACCACC-3' and catB-R-5'ATACGCGGTCACCTTCCTG-3', Tetracycline (tetB) with primer set tetB-F-5'- CTGTCGCGGCATCGGTCAT-3' and tetB-R-5'- CAGGTAAAGCGATCCCACC-3', Trimethoprim (dfrA12, drfA1) with primer sets drfA12-F-5'- TTCGCAGACTCACTGAGGG-3' and drfA12-R-5'- CGGTTGAGACAAGCTCGAAT-3', and drfA1-F-5'- CAATGGCTGTTGGTTGGAC3'and drfA1-R-5'- CCGGCTCGATGTCTATTGT-3', Spectinomycin (aadA2) with primer set aadA2-F-5'CATTGAGCGCCATCTGGAAT-3' and aadA2-R-5'ACATTTCGCTCATCGCCGGC-3' (Chuanchuen et al., 2008), Gentamicin (aadB) with primer set aadB-F-5'CTAGCTGCGGCAGATGAGC-3' and aadB-R-5'CTCAGCCGCCTCTGGGCA-3', Streptomycin(strB) with primer set strB-F-5'- GCGGACACCTTTTCCAGCCT-3' and strB-R.5'- TCCGCCATCTGTGCAATGCG-3' and Sulfamethoxazole (sul2) with primer set sul2-F-5'GCGCAGGCGCGTAAGCTGAT-3', and sul2-R-5'CGAAGCGCAGCCGCAATTC-3' (Chuanchuen \& Padungtod, 2009). A colony of Salmonella from each isolate was seeded in tryptone soy agar (TSA), and incubated for $24 \mathrm{hr}$ at $37^{\circ} \mathrm{C}$. Bacterial cells were collected, washed with PBS, pelleted into a $1.5 \mathrm{~mL}$ Eppendorf tube and total DNA was extracted using the phenol-chloroform-isoamyl alcohol method (Sambrook \& Russell, 2001). Bacterial DNA was diluted in $100 \mu \mathrm{L}$ $1 \times$ TE buffer and used as template in the PCR mixture to amplify the antibiotic resistance genes.

\section{Polymerase chain reaction, PCR}

The PCR was carried out in a total volume of $25 \mu \mathrm{L}$ containing $1 \mu \mathrm{L}$ of template DNA, $1 \mu \mathrm{L}$ of forward and $1 \mu \mathrm{L}$ of reverse primers (Invitrogen ${ }^{\mathrm{TM}}$, Thermo Fisher Scientific Inc.), $0.5 \mu \mathrm{L}$ of AccuprimeTaq polymerase, $2.5 \mu \mathrm{L}$ of $10 \times$ buffer, $2.5 \mu \mathrm{L}$ of $\mathrm{MgSO}_{4}$, and 16.5 $\mu \mathrm{L}$ of nuclease free water was also added. PCR was performed in a BIO-RAD T100'TM thermal cycler after an initial denaturation step of 1 minute at $94^{\circ} \mathrm{C}$, 35 cycles of amplification were performed. Each cycle consisted of the following steps: $60 \mathrm{~s}$ at $94^{\circ} \mathrm{C}$ (denaturation), $30 \mathrm{~s}$ at $55^{\circ} \mathrm{C}$ (primer annealing), and $30 \mathrm{~s}$ at $68^{\circ} \mathrm{C}$ (extension), followed by $7 \mathrm{~min}$ at $68^{\circ} \mathrm{C}$ for final extension. Salmonella Typhimurium (ATCC 14028) was used as a positive control, whereas the negative control did not contain DNA template. The reaction mixture was mixed with 2,5 $\mu \mathrm{L} 10 \times$ gel loading buffer and then resolved by electrophoresis on $2 \%$ agarose gel with 100 bp DNA ladder. The reaction products were stained with ethidium bromide and visualized under the UV light by using an ENDURO'M GDS (Labnet International, Inc.), GEL documentation system.

\section{Statistical Analysis}

Associations between phenotypic and genotypic antibiotic resistance in Salmonella were established by a Spearman correlation test (GraphPad Prism ${ }^{\circledR} 5.03$ version software).

\section{RESULTS}

All 47 Salmonella isolates (100\%) were resistant to five antibiotics belonging to Aminoglycosides (AMK, GEN, TOB) and Cephalosporin (FOX and CFZ) classes (Table 1). In total $57.4 \%$ of Salmonella isolates were resistant to Tetracycline (27/47), and $53.19 \%$ (25/47) were resistant to Ampicillin. At least $42.35 \%$ (20/47) of Salmonella isolates were found to be MDR strains that showed resistance to eleven (AMP, AMK, GEN, TOB, CFZ, FOX, NIT, SXT, TET, CIP and ENR) or more antibiotics belonging to seven antibiotic classes (Aminoglycosides, Penicillin, Cephalosporin, Nitrofurans, Sulfonamides/Trimethoprim, Tetracycline and quinolones). Salmonella isolates also exhibited resistance to phenicols $\mathrm{CHL}$ and FFC at a frequency of $6.38 \%$ (3 isolates). All Salmonella isolates were susceptible to ATM, FEP, ETP, IPM, MEM, TZP, TIM, NOR and FOF.

All isolates of S. Paratyphi B (36.17\%), S. Heidelberg, S. Typhimurium S. Muenster, and S. Hvittingfoss were classified as MDR strains with resistance to three or more antibiotics classes. One isolate of $S$. Typhimurium (UT-STm14018), one S. Paratyphi B (UT-SPb14010) and one S. Hvittingfoss (UT-SHv14023) showed resistance to phenicols and S. Heidelberg, S. Skansen, S. Schwarzengrund, S. Budapest and all S. Paratyphi B isolates showed resistance to ENR (Table 1).

The genotypic analysis showed the presence of a number of genes associated with antibiotic resistance such as blaTEM in $100 \%$ of Salmonella isolates, aadB in 41 out of 47 isolates (87.2\%), strB in $70.2 \%$, sul 2 in $57.4 \%, d r f A 1$ in $51 \%$, tet $B$ in $42.5 \%$ and aad $A 2$ in $38.2 \%$. The cat $B$ gene that is known to confer resistance to phenicols was present in the same Salmonella isolates $(100 \%)$ that showed phenotypic resistance by the Kirby-Bauer method. Most of the Salmonella isolates exhibited MDR genotype and none of isolates amplified the sequence drfA1 2 (Table 2). A good Spearman correlation coefficient between the phenotype and genotype was found for Chloramphenicol $(r=1.00)$, Gentamicin ( $r=0.94)$, Trimethoprim $(r=0.68)$ and 
Table 1 - Phenotypic and genotypic antibiotic resistance patterns of Salmonella serovars isolated from chicken meat sold at Ibague, Colombia (February-May 2014).

\begin{tabular}{|c|c|c|c|c|}
\hline Salmonella code & Group & Serovar/(formula) & Phenotype of antibiotic resistance ${ }^{(a)}$ & Genotype of antibiotic resistance \\
\hline UT-SNp14001 & $\mathrm{C} 2$ & Newport $(6,8: e, h: 1,2)$ & AMK, CFZ, FOX, GEN, TOB. & Bla TEM, Sul 2, aadB, drfA1 \\
\hline UT-SSk14002 & $\mathrm{C} 2$ & Skansen $(6,8: b: 1,2)$ & AMK, CFZ, FOX, CIP, GEN, NIT, TET, TOB, SXT, ENR. & Bla TEM, Str B, tet B, Sul 2, aadB, aadA2, drfA1 \\
\hline UT-SKa14003 & E1 & Kalina $(3,10: b: 1,2)$ & AMK, CFZ, FOX, GEN, TOB. & Bla TEM, aadB, aadA2, sul 2, drfA1 \\
\hline UT-SSc14004 & B & Schwarzengrund $(1,4,12,27: \mathrm{d}: 1,7)$ & AMK, CFZ, FOX, CIP, GEN, NIT, TET, TOB, SXT, ENR. & Bla TEM, tet B, Sul 2, Str B, aadB, drfA1 \\
\hline UT-SPb14005 & B & Parathyphi $B(1,4,5,12: b: 1,2)$ & AMK, AMP, SAM, CFZ, FOX, CRO, CIP, GEN, NIT, TET, TOB, SXT, ENR. & Bla TEM, tet B, Sul 2, aadB, aadA2, Str B, drfA1 \\
\hline UT-SPb14006 & B & Parathyphi B $(1,4,5,12: b: 1,2)$ & AMK, AMP, SAM, CFZ, FOX, CRO, CIP, GEN, NIT, TET, TOB, SXT, ENR. & Bla TEM, tet B, Sul 2,aadB, Str B, aadA2, drfA1 \\
\hline UT-SPb14007 & B & Parathyphi $B(1,4,5,12: b: 1,2)$ & AMK, AMP, SAM, CFZ, FOX, CRO, CIP, GEN, NIT, TET, TOB, SXT, ENR. & Bla TEM, tet B Sul 2, Str B, aadA2, aadB, drfA1 \\
\hline UT-SPb14008 & B & Parathyphi B $(1,4,5,12: b: 1,2)$ & AMK, AMP, SAM, CFZ, FOX, CRO, CIP, GEN, NIT, TOB, SXT, ENR. & Bla TEM, sul 2, aadB, aadA2, Str B, drfA1 \\
\hline UT-SPb14009 & B & Parathyphi $B(1,4,5,12: b: 1,2)$ & AMK, AMP, SAM, CFZ, FOX, CRO, CIP, GEN, NIT, TOB, SXT, ENR. & Bla TEM, sul 2, aadA2, Str B, aadB, drfA1 \\
\hline UT-SPb14010 & B & Parathyphi B $(1,4,5,12: b: 1,2)$ & AMK, AMP, SAM, CFZ, FOX, CRO, CIP, GEN, NIT, TET, TOB, SXT, ENR, FFC. & Bla TEM tet B, Sul 2, Str B, aadA2, aadB, catB, drfA1 \\
\hline UT-SPb14011 & B & Parathyphi B $(1,4,5,12: b: 1,2)$ & AMK, AMP, SAM, CFZ, FOX, CRO, CIP, GEN, NIT, TET, TOB, SXT, ENR. & Bla TEM, tet B, Sul 2, Str B, aadA2, aadB, drfA1 \\
\hline UT-SMh14012 & $\mathrm{C} 2$ & Manhattan $\quad(6,8: \mathrm{d}: 1,5)$ & AMK, CFZ, FOX, GEN, TOB. & Bla TEM, Sul 2, aadB, Str B, drfA1 \\
\hline UT-SPb14013 & B & Parathyphi B $(1,4,5,12: b: 1,2)$ & AMK, AMP, SAM, CFZ, FOX, CRO, CIP, GEN, NIT, TET, TOB, SXT, ENR. & Bla TEM, tet B, Sul 2, Str B, aadA2, aadB, drfA1 \\
\hline UT-SBr14014 & C1 & Braenderup $(6,7,14: e, h: e, n, z, 15)$ & AMK, CFZ, FOX, GEN, TOB. & Bla TEM, Sul 2, aadB, Str B \\
\hline UT-SBr14015 & C1 & Braenderup $(6,7,14: e, h: e, n, z, 15)$ & AMK, CFZ, FOX, GEN, TOB. & Bla TEM, tet B, Sul 2, aadB, Str B, \\
\hline UT-SBm14016 & $\mathrm{C2}$ & Bovismorbificans $(6,8,20: r: 1,5)$ & AMK, CFZ, FOX, GEN, TOB. & Bla TEM Sul 2, aadB, \\
\hline UT-STm14017 & B & Typhimurium (1,4,12:i:-) & AMK, CFZ, FOX, GEN, TET, TOB, CHL, FFC. & Bla TEM, tet B, Sul 2, addB, Str B, catB \\
\hline UT-STm14018 & B & Typhimurium (1,4,12:i:-) & AMK, CFZ, FOX, GEN, TET, TOB, & Bla TEM, Sul 2, aadB, \\
\hline UT-SOt14019 & C1 & Othmarschen $(6,7: \mathrm{g}, \mathrm{m}, \mathrm{t}:-)$ & AMK, CFZ, FOX, GEN, TOB. & Bla TEM, Sul 2, aadB \\
\hline UT-SPb14020 & B & Parathyphi B $(1,4,5,12: b: 1,2)$ & AMK, AMP, SAM, CFZ, FOX, CRO, CIP, GEN, NIT, TET, TOB, SXT, ENR. & Bla TEM, tet B, Sul 2, Str B, aadB, drfA1 \\
\hline UT-SNp14021 & C2 & Newport $\quad(6,8: e, h: 1,2)$ & AMK, CFZ, FOX, GEN, TOB. & Bla TEM, Sul 2, Str B aadB, drfA1 \\
\hline UT-SPb14022 & B & Parathyphi B $(1,4,5,12: b: 1,2)$ & AMK, AMP, SAM, CFZ, FOX, CRO, CIP, GEN, NIT, TET, TOB, SXT, ENR. & Bla TEM, tet B, Sul 2, Str B, aadB, drfA1 \\
\hline UT-SHv14023 & 1 & Hvittingfoss (16:b:e, $n, x)$ & AMK, CFZ, FOX, GEN, TOB, FFC. & Bla TEM Sul 2, aadB, catB \\
\hline UT-SHv14024 & 1 & Hvittingfoss (16:b:e,n,x) & AMK, CFZ, FOX, GEN, TOB, & Bla TEM, Sul 2, aadB, \\
\hline UT-SHe14025 & B & Heidelberg $(1,4,12: r: 1,2)$ & AMK, AMP, CFZ, FOX, CIP, GEN, NIT, TET, TOB, SXT, ENR. & Bla TEM, tet B, Sul 2, Str B, aadB, \\
\hline UT-SHv14026 & I & Hvittingfoss (16:b:e,n,x) & AMK, CFZ, FOX, GEN, TOB, & Bla TEM, aadA2, aadB, \\
\hline UT-SHe14027 & B & Heidelberg $(1,4,12: r: 1,2)$ & AMK, AMP, CFZ, FOX, CIP, GEN, NIT, TET, TOB, SXT, ENR. & Bla TEM. Str B \\
\hline UT-SHv14028 & I & Hvittingfoss (16:b:e,n,x) & AMK, CFZ, FOX, GEN, TOB, & Bla TEM, aadB, \\
\hline UT-SHv14029 & 1 & Hvittingfoss (16:b:e,n,x) & AMK, CFZ, FOX, GEN, TOB, & Bla TEM, aadB, \\
\hline UT-SHv14030 & 1 & Hvittingfoss (16:b:e,n,x) & AMK, CFZ, FOX, GEN, TOB, & Bla TEM \\
\hline UT-SHv14031 & 1 & Hvittingfoss (16:b:e,n,x) & AMK, CFZ, FOX, GEN, TOB, & Bla TEM \\
\hline UT-SHv14032 & 1 & Hvittingfoss (16:b:e,n,x) & AMK, CFZ, FOX, GEN, TOB, & Bla TEM \\
\hline UT-SHv14033 & 1 & Hvittingfoss (16:b:e,n,x) & AMK, CFZ, FOX, GEN, TOB, & Bla TEM \\
\hline UT-SPb14034 & B & Parathyphi $B(1,4,5,12: b: 1,2)$ & AMK, AMP, SAM, CFZ, FOX, CRO, CIP, GEN, NIT, TET, TOB, SXT, ENR. & Bla TEM, Str B, drfA1 \\
\hline UT-SMu14035 & E1 & Muenster $(3,10: e, h: 1,5)$ & AMK, AMP, CFZ, FOX, GEN, TET, TOB. & Bla TEM, Str B, addB, aadA2, \\
\hline UT-SMu14036 & E1 & Muenster $(3,10: e, h: 1,5)$ & AMK, AMP, CFZ, FOX, GEN, TET, TOB. & Bla TEM, Sul 2, Str B, aadB \\
\hline UT-SMu14037 & E1 & Muenster $(3,10: e, h: 1,5)$ & AMK, AMP, CFZ, FOX, GEN, TET, TOB. & Bla TEM, Str B, aadB \\
\hline UT-SMu14038 & E1 & Muenster (3,10:e,h:1,5) & AMK, AMP, CFZ, FOX, GEN, TET, TOB. & Bla TEM, Sul 2, Str B, aadB \\
\hline UT-SMu14039 & E1 & Muenster $(3,10: e, h: 1,5)$ & AMK, AMP, CFZ, FOX, GEN, TET, TOB. & Bla TEM, Sul 2, Str B, aadB \\
\hline UT-SKa14040 & E1 & Kalina $(3,10: b: 1,2)$ & AMK, CFZ, FOX, GEN, TOB. & Bla TEM, tet B, Str B, aadB, drfA1 \\
\hline UT-SPb14041 & B & Parathyphi B $(1,4,5,12: b: 1,2)$ & AMK, AMP, SAM, CFZ, FOX, CRO, CIP, GEN, NIT, TET, TOB, SXT, ENR. & Bla TEM, tet B, Str B, aadB, drfA1 \\
\hline UT-SPb14042 & B & Parathyphi B $(1,4,5,12: b: 1,2)$ & AMK, AMP, SAM, CFZ, FOX, CRO, CIP, GEN, NIT, TET, TOB, SXT, ENR. & Bla TEM, tet B, Str B, aadA2, aadB, drfA1 \\
\hline UT-SPb14043 & B & Parathyphi $B(1,4,5,12: b: 1,2)$ & AMK, AMP, SAM, CFZ, FOX, CRO, CIP, GEN, NIT, TET, TOB, SXT, ENR. & Bla TEM, tet B, Str B, aadA2, aadB, drfA1 \\
\hline UT-SPb14044 & B & Parathyphi B $(1,4,5,12: b: 1,2)$ & AMK, AMP, SAM, CFZ, FOX, CRO, CIP, GEN, NIT, TET, TOB, SXT, ENR. & Bla TEM, tet B, Str B, aadA2, aadB, drfA1 \\
\hline UT-SBu14045 & B & Budapest $(4,12: g, t:-)$ & AMK, AMP, SAM, CFZ, FOX, CRO, CIP, GEN, NIT, TET, TOB, ENR. & Bla TEM, tet B, Str B, aadA2, aadB, drfA1 \\
\hline UT-SPb14046 & B & Parathyphi B $(1,4,5,12: b: 1,2)$ & AMK, AMP, SAM, CFZ, FOX, CRO, CIP, GEN, NIT, TET, TOB, SXT, ENR. & Bla TEM, tet B, Str B, aadA2, aadB, drfA1 \\
\hline UT-SPb14047 & B & Parathyphi $B(1,4,5,12: b: 1,2)$ & AMK, AMP, SAM, CFZ, FOX, CRO, CIP, GEN, NIT, TET, TOB, SXT, ENR. & Bla TEM, Str B, aadA2, aadB, drfA1 \\
\hline
\end{tabular}

a). Antibiotic resistance patterns of Salmonella determined by the disc-diffusion and microdilution assays. AMK, Amikacin; AMP, Ampicillin; SAM, Ampicillin-Sulbactam; CFZ, Cefazoline; FOX, Cefoxitin; CRO, Ceftriaxone; CIP, Ciprofloxacin; GEN, Gentamicin; NIT, Nitrofurantoin; TET, Tetracycline; TOB, Tobramycin; CHL, Chloramphenicol; FFC, Florfenicol; SUL, Sulfamethoxazole; SXT, Trimethoprim-Sulfamethoxazole, ENR, Enrofloxacin.

Tetracycline $(r=0.66)$ and lower coefficients for Ampicillin ( $r=0.57)$ and Sulfamethoxazole $(r=0.30)$. The majority of the PCR products were higher in size than those reported in Salmonella isolated in Thailand (Chuanchuen \& Padungtod, 2009). For example, the expected size of catB was $461 \mathrm{bp}$, however, there were multiple bands with one prominent band of about 800 $\mathrm{bp}$ and a similar situation was found with the $\mathrm{aadB}$ gene (expected band at 300bp) that showed a PCR product of about 800 bp. All other antibiotic resistance genes showed PCR products with similar size to the expected band.

\section{DISCUSSION}

The results of this study indicates that Salmonella isolated from chicken carcasses sold at Ibague Colombia during February to May 2014 showed a broad spectrum 
Table 2 - Frequency of antibiotic resistance genes detected in Salmonella spp., isolated from chicken meat sold at Ibague, Colombia (February-May 2014).

\begin{tabular}{|c|c|c|c|c|}
\hline Antibiotic & Gene name / & PCR product (bp) & $\mathrm{N}^{\circ}$ of positives $(\%)$ & Serovars(a) (Number) \\
\hline Ampicillin & bla TEM & $(608)$ & $47(100 \%)$ & $\begin{array}{l}\text { ParB (17), Hvi (9), Mue (5), Typ (2), New (2), Hei (2), Bra (2), } \\
\text { Kal (2), Bov (1), Bud (1), Man (1), Oth (1), Sch (1), Ska (1). }\end{array}$ \\
\hline Gentamicin & $\mathrm{aadB}$ & $(300)$ & $41(87.2 \%)$ & $\begin{array}{l}\text { ParB (16), Hvi (5), Mue (5), Typ (2), New (2), Hei (1), Bra (2), } \\
\operatorname{Kal}(2), \operatorname{Bov}(1), \operatorname{Bud}(1), \operatorname{Man}(1), \operatorname{Oth}(1), \operatorname{Sch}(1), \operatorname{Ska}(1) .\end{array}$ \\
\hline Streptomycin & $s t r B$ & $(621)$ & $33(70.2 \%)$ & $\begin{array}{l}\text { ParB (17), Mue (5), Typ (1), New (1), Hei (2), Bra (2), Kal (1), } \\
\text { Bud (1), Man (1), Sch (1), Ska (1). }\end{array}$ \\
\hline Sulfamethoxazole & sul2 & (514) & $27(57.4 \%)$ & $\begin{array}{l}\operatorname{ParB}(9), \operatorname{Hvi}(2), \operatorname{Mue}(3), \operatorname{Typ}(2), \operatorname{New}(2), \operatorname{Hei}(1), \operatorname{Bra}(2) \text {, } \\
\text { Kal (1), Bov (1), Man (1), Oth (1), Sch (1), Ska (1). }\end{array}$ \\
\hline Tetracycline & tetB & $(615)$ & $20(42.5 \%)$ & ParB (13), Typ (1), Hei (1), Bra (1), Kal (1), Bud (1), Sch (1), Ska (1). \\
\hline Spectinomycin & aadA2 & $(500)$ & $18(38.2 \%)$ & $\operatorname{ParB}(13), \operatorname{Hvi}(1), \operatorname{Mue}(1), \operatorname{Kal}(1), \operatorname{Bud}(1), \operatorname{Ska}(1)$. \\
\hline Chloramphenicol & cat B & (461) & $3(6.38 \%)$ & $\operatorname{ParB}(1), \operatorname{Typ}(1), \operatorname{Hvi}(1)$ \\
\hline \multirow[t]{2}{*}{ Trimethoprim } & drfA1 & (254) & $24(51.0 \%)$ & $\operatorname{ParB}(17), \operatorname{New}(2), \operatorname{Kal}(2), \operatorname{Man}(1), \operatorname{Sch}(1)$, Ska (1). \\
\hline & drfA12 & (330) & $0(0 \%)$ & \\
\hline
\end{tabular}

a) ParB, Paratyphi B; Hvi, Hvittingfoss; Mue, Muenster; Typ, Typhimurium; New, Newport; Hei, Heidelberg; Bra, Branderup; Kal, Kalina; Bov, Bovismorbificans; Bud., Budapest; Man, Manhattan; Oth, Othmarschen; Sch, Schwarzengrund; Ska, Skansen.

of antibiotic resistance when compared with isolates from other meat sources (Mąka et al., 2014) or clinical isolates (Vaz et al., 2010). A variety of Salmonella serovars resistant to multiple antibiotic classes were present in chicken meat samples and a high percentage $(42.35 \%)$ of Salmonella showed multi-drug resistance (MDR) by the phenotypic method, among them, all S. Paratyphi B isolates and S. Heildelberg were resistant to 7 different classes of antibiotics, whereas S. Muenster and S. Typhimurium were resistant to 4 different classes of antibiotics. These results are similar to those reported in Salmonella Paratyphi B isolated from broiler farms in Cundinamarca and Santander, where this serovar was found to be resistant to up to 15 antimicrobials (range 9-15) (Donado-Godoy et al., 2012), of which resistance to NIT, SXT, TET, CIP and ENR can be identified as a common resistance pattern that is consistent at least in part with the usage of tetracycline, trimethoprim and quinolones in the regional poultry industry (based on author's survey). Thus, the choice of fluoroquinolones in treatment of severe infections by Salmonella could be seriously impeded, as it has been noted by others (Ricke \& Calo, 2015). The percentage of MDR Salmonella isolated in this study was lower than that reported in Salmonella from different meats (70\%) in China, where 252 out of 359 isolates showed MDR phenotypes to at least three classes of antibiotics and the chicken isolates had the higher resistance (80\%) rates (Yang et al., 2010).

Salmonella Enteritidis and S. Typhimurium are the most widespread serovars and can be isolated from surface water, meat and poultry (Jokinen et al., 2015; Yang et al., 2010), and constitute the principal serovars responsible for human and animal disease (MPS, 2011; Stevens et al., 2009), however, our previous study reported that Salmonella Enteritidis was not isolated from chicken meat sold at stores and supermarkets of Ibague, and instead Salmonella Paratyphi B var Java was the most prevalent serovar (36.17\%) followed by Hvittingfoss (19.15\%), and Muenster (10.64\%) (Rodriguez et al., 2015b). S. Paratyphi B variant Java $(76.4 \%)$ and S. Heildelberg $(22.7 \%)$ were the most prevalent serovars isolated from broiler farms in two distinct regions (Cundinamarca and Santander) of Colombia (Donado-Godoy et al., 2012), thus, this serovar might be distributed in broiler farms across the country and its potential association with natural outbreaks of paratyphoid disease reported by the National Institute of Health (INS, 2015), is worthy of investigation. S. Paratyphi B var Java has been isolated from poultry from Netherlands (Van Pelt et al., 2003) and Germany (Dorn et al., 2001), from chicken viscera at two slaughter plants in the state of Zulia, Venezuela (Boscan et al., 2005), from chicken in Belgium (De Jong et al., 2014), and from breeders and broiler farms in Bangladesh (Barua et al., 2013). In Bangladesh, it was also isolated from blood of patients with clinically diagnosed enteric fever at similar proportions to $S$. Typhi but with higher resistance rates (Afroz et al., 2014), highlighting an increased risk upon its eventual transmission to human. Thus, whether the increased frequency of this serovar relies on changes in the population dynamics (Foley et al., 2011) of S. enterica serovars in broiler farms in Colombia is an issue that also needs further investigation.

The MDR resistance rate of Salmonella isolates in this study was higher than that reported in Salmonella from chicken carcasses $(n=123)$ and chicken meat in Italy, where $30.5 \%$ and $36 \%$ exhibited multi-drug resistance to AMP, SUL and TET, respectively (Bacci 
et al., 2012), however, in that study, authors may have used a limited number of antibiotic agents. Our results are also similar to the MDR Salmonella spp., reported in Southeast Asian countries such as Malaysia, Thailand and Vietnam where the resistance rate ranged between 21 to $75 \%$, and resistance to traditional antibiotics such as AMP, SUL and TET was high in Salmonella isolated from animals and foods of animal origin in Malaysia (resistance rate 22 - 49\%), Thailand (41 - 92\%), and Vietnam (17 - 68\%) (Van et al., 2012). The results may suggest that misuse or indiscriminate use of antimicrobials in poultry of the Tolima region is contributing to increase the antibiotic resistant strains of Salmonella.

S. Typhimurium was found to be resistant to eight antibiotics (AMK, GEN, TOB, TET, CZO, FOX, CHL, FFC), results similar to MDR $S$. Typhimurium isolated from human, chicken and cattle from Malaysia (Benacer et al., 2010), ducks from Malaysia (Adzitey et al, 2012), and from fresh raw chicken carcasses sold at retail in different markets in central Anatolia, Turkey (Yildirim et al., 2011). MDR S. Typhimurium and S. Branderup, and $S$. Muenster have been isolated from outbreaks of salmonellosis in Colombia (INS, 2014), suggesting a potential link between poultry and salmonellosis in this region. The MDR phenotype of S. Paratyphi $B, S$. Muenster, S. Typhimurium and S. Heildelberg isolated from chicken carcasses in this study indicate an increased risk and concern in the case of its eventual transmission to humans and suggest the need to search for those serovars in cases of diarrheal disease in the Tolima region.

The phenotypic MDR pattern of Salmonella showed partial correlation with the genotypic analysis. The blaTEM gene sequence was present in all Salmonella isolates $(100 \%)$, however it had low correlation ( $r=$ 0.577 ) with the phenotypic AMP resistance phenotype by the Spearman correlation test. In contrast, the presence of $\operatorname{aad} B(87 \%)$ gene that confers resistance to Gentamicin had a high correlation with this phenotypic antibiotic resistance $(r=0.94)$. Although the phenotypic analysis of Streptomycin resistance was not evaluated in this study, we found a high prevalence of the strB (70\%) gene that has been described to confer such antibiotic resistance (Brenner et al., 2013). Regarding to the phenotypic resistance to Sulfonamides/Trimethoprim (SXT), we found similar frequencies of the sul2 (57\%) and drfA 1 $(51 \%)$ genes that are associated with Sulfonamides and Trimethoprim resistance, respectively. The aadA2 $(25 \%)$ gene involved in Spectinomycin resistance was also frequently detected (Table 1). The differences between the resistance of Salmonella to some antibiotics assessed by the phenotypic methods and the resistance pattern obtained by PCR might be due to different antibiotic resistance genes that were not evaluated in this study because of financial constraints. In this regard, a number of antibiotic resistance genes had been described in Salmonella (Brenner et al., 2013; Chen et al., 2004). Thus, it is important to increase awareness of the potential impact of antibiotic resistant strains of Salmonella present in poultry products in the Tolima region and the need to increase funding to promote this research.

In conclusion, this study found that about $42 \%$ of Salmonella serovars isolated from chicken meat sold at stores and supermarkets of Ibague city were resistant to multiple classes of antibiotics by both phenotypic and genotypic tests and this data agree with the global health concern imposed by antibiotic resistant strains that may limit the choice of treatment of human infections. The results suggest various needs that include cooperation between the poultry industry, governmental and academic institutions to improve the surveillance of both Salmonella and its antibiotic resistance patterns in broiler farms and poultry products, to establish appropriate regulations and funding for antibiotic research, and to promote education and prudent use of antibiotics by poultry farmers.

\section{ACKNOWLEDGEMENTS}

This research was funded by grants from the Central Research Office of the University of Tolima to Noel Verjan García (Projects No. 970213 and No. 240214). The authors thank the owners of the stores that participated in the study, to Dr. Clemencia Fandiño for technical assistance in Salmonella isolation and Gisella Holguin for technical assistance in PCR.

\section{REFERENCES}

Adzitey F, Rusul G, Huda N. Prevalence and antibiotic resistance of Salmonella serovars in ducks, duck rearing and processing environments in Penang, Malaysia. Food Research International 2012;45(2):947-952. Available from: <http://doi.org/urn:doi:10.1016/j.foodres.2011.02.051>

Afroz $\mathrm{H}$, Hossain MM, Fakruddin M. A 6-year retrospective study of bloodstream Salmonella infection and antibiotic susceptibility of Salmonella enterica serovar Typhi and Paratyphi in a tertiary care hospital in Dhaka, Bangladesh. Tzu Chi Medical Journal 2014;26(2):73-78. Available from: http://doi.org/ urn:doi:10.1016/j.tcmj.2014.05.006.

Bacci C, Boni E, Alpigiani I, Lanzoni E, Bonardi S, Brindani F. Phenotypic and genotypic features of antibiotic resistance in Salmonella enterica isolated from chicken meat and chicken and quail carcasses. International Journal of Food Microbiology 2012;160(1):16-23. Available from: http://doi.org/ urn:doi:10.1016/j.ijfoodmicro.2012.09.014. 
Barua H, Biswas PK, Olsen KEP, Shil SK, Christensen JP, Majowicz S, et al. Molecular characterization of motile serovars of Salmonella enterica from breeder and commercial broiler poultry farms in Bangladesh. PLoS ONE 2013;8(3):e57811. Available from: http://doi.org/urn:doi:10.1371/ journal.pone.0057811.

Benacer D. Thong KL, Watanabe $\mathrm{H}$, Puthucheary SD. Characterization of drug resistant Salmonella enterica serotype Typhimurium by antibiograms, plasmids, integrons, resistance genes and PFGE. Journal of Microbiology and Biotechnology 2010;20(6):1042-1052. Available from: http://doi.org/ urn:doi:10.4014/jmb.0910.10028.

Boscan DLA, Arzálluz AM, Ugarte Cl, Sánchez D, Díaz D, Wittum TE, et al. Aislamiento de Salmonellas de importancia zoonótica en vísceras de pollos beneficiados en el estado Zulia, Venezuela. Revista Cientifica FCV-LUZ 2005;15(6):576-582.

Brenner G, Hall R, Fanning S, Schwarz S. Antimicrobial resistance in Salmonella. In: Barrow P, Methner U, editor. Salmonella in domestic animals. 2nd ed. Wallingford: CABl; 2013. p.120-135.

Brown-Jaque M, Calero-Cáceres W, Muniesa M. Transfer of antibioticresistance genes via phage-related mobile elements. Plasmid 2015;79:1-7. Available from: http://doi.org/ urn:doi:10.1016/j. plasmid.2015.01.001.

CDC. Reports of selected Salmonella outbreak investigations. Atlanta: Centers for Disease Control and Prevention; 2014. Available from: https://www.cdc.gov/salmonella/outbreaks.html .

Chen MH, Chiou CS, Chiang YC, Chen PH, Tsai SW, Tsen HY. Comparison of the pulsed field gel electrophoresis patterns and virulence profiles of the multidrug resistant strains of Salmonella enterica serovar Schwarzengrund isolated from chicken meat and humans in Taiwan. Food Research International 2012:45(2):978-983. Available from: http://doi.org/ urn:doi:10.1016/j.foodres.2011.01.039.

Chen S, Zhao S, White DG, Schroeder CM, Lu R, Yang $H$, et al. Characterization of multiple-antimicrobial-resistant Salmonella serovars isolated from retail meats. Applied and Environmental Microbiology 2004;70(1):1-7.

Chuanchuen R, Padungtod P. Antimicrobial resistance genes in Salmonella enterica isolates from poultry and swine in Thailand. The Journal of Veterinary Medical Science /Japanese Society of Veterinary Science 2009;71(10):1349-55. Available from: http://www.ncbi.nlm.nih.gov/ pubmed/19887742.

Chuanchuen R, Pathanasophon P, Khemtong S, Wannaprasat W, Padungtod P. Susceptibilities to antimicrobials and disinfectants in Salmonella isolates obtained from poultry and swine in Thailand. The Journal of Veterinary Medical Science / Japanese Society of Veterinary Science 2008;70(6):595-601.

Claudi B, Spröte P, Chirkova A, Personnic N, Zankl J, Schürmann N, et al. Phenotypic variation of Salmonella in host tissues delays eradication by antimicrobial chemotherapy. Cell 2014;158(4):722-733. Available from: http://doi.org/ urn:doi:10.1016/j.cell.2014.06.045

CLSI. Performance Standards for Antimicrobial Disk Susceptibility Tests:Approved Standard-Twelft Edition 2015. Wayne PA.

De Jong A, Smet A, Ludwig C, Stephan B, De Graef E, Vanrobaeys M, et al. Antimicrobial susceptibility of Salmonella isolates from healthy pigs and chickens (2008-2011). Veterinary Microbiology 2014;171(34):298-306. Available from: http://doi.org/ urn:doi:10.1016/j. vetmic.2014.01.030

Dekker JP, Frank KM. Salmonella, Shigella, and Yersinia. Clinics in Laboratory Medicine 2015;35(2):225-246. Available from: http://doi. org/10.1016/j.cll.2015.02.002
Diard M, Sellin ME, Dolowschiak T, Arnoldini M, Ackermann M, Hardt WD. Antibiotic treatment selects for cooperative virulence of Salmonella typhimurium. Current Biology 2014;24(17):2000-2005. Available from: http://doi.org/ urn:doi:10.1016/j.cub.2014.07.028

Díaz Osorio MA, Díaz Guevara PL, Rodríguez Cárdenas EC, Montaño Valencia LA, Medina Alfonso IM, Patiño González GI, et al. Caracterización fenotípica y genotípica de Salmonella Typhimurium variante 5- asociada a un brote de enfermedad transmitida por alimentos en el municipio de Paz de Río , Boyacá , 2010. IATREIA 2014;27(1):23-30.

Donado-Godoy P, Gardner I, Byrne Ba, Leon M, Perez-Gutierrez E, Ovalle $M V$, et al. Prevalence, risk factors, and antimicrobial resistance profiles of Salmonella from commercial broiler farms in two important poultry-producing regions of Colombia. Journal of Food Protection 2012;75(5):874-883. Available from: http://doi.org/ urn:doi:10.4315/0362-028X.JFP-11-458

Dorn C, Schroeter A, Miko A, Protz D, Helmuth R. Increasing number of Salmonella paratyphi B isolates from slaughtered poultry sent in to the national Salmonella reference laboratory. Berliner und Münchener Tierärztliche Wochenschrift 2001;114(5-6):179-183. Available from: http://europepmc.org/abstract/med/11413710

Foley SL, Nayak R, Hanning IB, Johnson TJ, Han J, Ricke SC. Population dynamics of Salmonella enterica serotypes in commercial egg and poultry production. Applied and environmental Microbiology 2011;77(13):4273-4279. Available from: http://doi.org/ urn:doi:10.1128/AEM.00598-11

Grimont PA, Weill FX. Antigenic Formulae of the Salmonella serovars. 9th ed. Paris: WHO; 2007.

Hendriksen RS, Vieira AR, Karlsmose S, Lo Fo Wong DMA, Jensen $A B$, Wegener $H C$, et al. Global monitoring of Salmonella serovar distribution from the World Health Organization Global Foodborne Infections Network Country Data Bank:results of quality assured laboratories from 2001 to 2007. Foodborne Pathogens and Disease 2011;8(8):887-900. Available from: http://doi.org/ urn:doi:10.1089/ fpd.2010.0787

INS - Instituto Nacional de Salud. Grupo de microbiología. serotipos y patrones de susceptibilidad antimicrobiana. Salmonella spp a 30 de Diciembre de 2014. Bogotá; 2014. Available from: http://www.ins.gov. $\mathrm{co} /$ ? idcategoria $=1738$

INS - Instituto Nacional de Salud. Boletín Epidemiológico Semana 21 de 2015. Dirección de vigilancia y análisis del riesgo en Salud Pública. Bogotá 2015. Available from: http://www.ins.gov.co/?idcategoria=1738

Jokinen CC, Koot J, Cole L, Desruisseau A, Edge TA, Khan IUH, et al. The distribution of Salmonella enterica serovars and subtypes insurface water from five agricultural regions across Canada. Water Research 2015;76:120-131. Available from: http://doi.org/ urn:doi:10.1016/j. watres.2015.02.038

Majowicz SE, Musto J, Scallan E, Angulo FJ, Kirk M, O'Brien SJ, et al. International Collaboration on enteric disease "burden of illness" studies. the global burden of nontyphoidal Salmonella gastroenteritis. Clinical Infectious Diseases 2010;50(6):882-889. Available from: http:// doi.org/ urn:doi:10.1086/650733

Mąka Ł, Maćkiw E, Ścieżyńska H, Pawłowska K, Popowska M. Antimicrobial susceptibility of Salmonella strains isolated from retail meat products in Poland between 2008 and 2012. Food Control 2014;36(1):199-204. Available from: http://doi.org/urn:doi:10.1016/j.foodcont.2013.08.025

Mercado M, Avila J, Rey M, Montoya M, Gamboa A, Carrascal AK, et al. Brotes por Salmonella spp., Staphylococcus aureus y Listeria monocytogenes asociados al consumo de pollo. Revisión sistemática de la literatura. Biomédica 2012;32(3):375-385. Available from: http:// doi.org/ urn:doi:10.7705/biomedica.v32i3.697 
MPS - Ministerio de Protección Social . Perfil de riesgo Salmonella spp. (no tifoideas) en pollo etero y en piezas. Bogotá. Unidad de Evaluación de Riesgos para la inocuidad de los Alimentos UERIA. Bogotá: Instituto Nacional de Salud; 2011. Available from: http://www.ins. gov.co/lineas-de-accion/investigacion/ueria/Publicaciones/PERFIL \%20 SALMONELLA\%20SPP.pdf

OIE. World Organization for animal health. Salmonellosis. In: OIE manual of diagnostic tests and vaccines for terrestrial animals. Paris; 2012. Available from: <http://www.oie.int/doc/ged/D12009.PDF

Ricke SC, Calo JR. Antibiotic resistance in Pathogenic Salmonella. In: Chem CY, Yanm X, Jackson CR. Antimicrobial resistance and food safety. London: Academic Press; 2015. p.37-53. Available from: http://doi. org/ urn:doi:10.1016/B978-0-12-801214-7.00003-X

Rodriguez J, Rondón I, Verjan N. Serotypes of Salmonella in broiler carcasses marketed at ibague, Colombia. Revista Brasileira de Ciência Avícola 2015a;17(4):545-552. Available from: http://doi.org/ urn:doi:10.1590/1516-635X1704545-552

Rodriguez R, Fandiño C, Donado P, Guzmán L, Verjan, N. Characterization of Salmonella from commercial egg-laying hen farms in a central region of Colombia. Avian Diseases 2015b;59(57):59-63. Available from: http://www.aaapjournals.info/doi/pdf/10.1637/10873-052714-Reg

Sambrook J, Russell D. Molecular cloning: a laboratory manual. 3rd ed. J.F. New York: Cold Spring Harbor Laboratory Press; 2001.

Sanderson KE, Nair S. Taxonomy and species concepts in the genus Salmonella. In: Barrox PA, Methner $Y$, editors. Salmonella in domestic animals. Wallingford: Cabi; 2013. p.1-19. Available from: http://doi. org/ urn:doi:10.1079/9781845939021.0001
Stevens MP, HumphreyTJ, Maskell DJ. Molecular insights into farm animal and zoonotic Salmonella infections. Philosophical Transactions of the Royal Society of London. Series B, Biological Sciences 2009;364(1530):2709_ 2723. Available from: http://doi.org/ urn:doi:10.1098/rstb.2009.0094

Van Pelt W, van der Zee H, Wannet WJB, van de Giessen AW, Mevius DJ, Bolder NM, et al. Explosive increase of Salmonella Java in poultry in the Netherlands: consequences for public health. Euro Surveillance 2003;8(2):31-35

Van TTH, Nguyen HNK, Smooker PM, Coloe PJ. The antibiotic resistance characteristics of non-typhoidal Salmonella enterica isolated from food-producing animals, retail meat and humans in South East Asia. International Journal of Food Microbiology 2012;154(3):98-106. Available from: http://doi.org/ urn:doi:10.1016/j. ijfoodmicro.2011.12.032

Vaz CSL, Streck F, Michael GB, Marks FS, Rodrigues DP, Dos Reis EMF, et al. Antimicrobial resistance and subtyping of Salmonella enterica subspecies enterica serovar Enteritidis isolated from human outbreaks and poultry in southern Brazil. Poultry Science 2010;89(7):1530-1536. Available from: http://doi.org/ urn:doi:10.3382/ps.2009-00453

Yang B, Qu D, Zhang X, Shen J, Cui S, Shi Y, et al. Prevalence and characterization of Salmonella serovars in retail meats of marketplace in Shaanxi, China. International Journal of Food Microbiology 2010;141(1-2):63-72. Available from: http://doi.org/ urn:doi:10.1016/j. ijfoodmicro.2010.04.015

Yildirim Y, Gonulalan Z, Pamuk S, Ertas N. Incidence and antibiotic resistance of Salmonella spp. on raw chicken carcasses. Food Research International 2001;44(3):725-728. Available from: http://doi.org/ urn:doi:10.1016/j.foodres.2010.12.040 